Schmerz 2022 $\cdot 36: 406-421$

https://doi.org/10.1007/s00482-021-00608-8

Angenommen: 21. Oktober 2021

Online publiziert: 5 . Januar 2022

(c) Der/die Autor(en) 2022

\section{Effekte von präoperativer neurobiologischer Edukation auf das postoperative Outcome}

\author{
Ein systematisches Review
}

\author{
Karolin von Korn ${ }^{1,2} \cdot$ Thomas Weiss ${ }^{3} \cdot$ Harry von Piekartz ${ }^{2}$ \\ 'Lubinus Aktiv GmbH, Kiel, Deutschland \\ ${ }^{2}$ Fakultät Wirtschafts- und Sozialwissenschaften, Physiotherapie und Rehabilitationswissenschaften, \\ Hochschule Osnabrück, Osnabrück, Deutschland \\ ${ }^{3}$ Lehrstuhl für Klinische Psychologie, Friedrich-Schiller-Universität Jena, Jena, Deutschland
}

\title{
Zusammenfassung
}

Hintergrund: Schmerz hat einen entscheidenden Einfluss auf die humane Lebensqualität. Allein eine Wissensvergrößerung über neurobiologische Vorgänge kann das subjektive Schmerzempfinden sowie psychometrische Variablen positiv beeinflussen. Es gibt verschiedene Formen der präoperativen Patientenedukation, welche u.a. zum Ziel haben, den postoperativen Schmerz zu erklären. Laut der aktuellen Literatur liegt einer präoperativen biomedizinischen Edukation eine geringe Evidenz zugrunde. Sie kann das präoperative Angst- und Stresslevel der Patienten steigern, was sich negativ auf das postoperative Outcome auswirkt. Im Gegensatz zur biomedizinischen Edukation betrachtet das neurobiologische Verständnis den postoperativen Schmerz unter den Gesichtspunkten der Plastizität des Nervensystems und bezieht Sensibilisierungsprozesse im zentralen und peripheren Nervensystem mit ein.

Ziel: Systematische Untersuchung von Kurz- und Langzeiteffekten einer neurobiologischen (Schmerz-)Edukation bei Patienten vor einer Wirbelsäulenoperation Material und Methoden: Bei der Literaturrecherche wurde nach dem $\mathrm{PI}(\mathrm{C}) \mathrm{O}$ (Population Intervention Comparison Outcome)-Schema in den medizinischen Datenbanken gesucht. 83 Artikel kamen in die engere Auswahl. Entsprechend den Ein- und Ausschlusskriterien konnten letztendlich neun Artikel eingeschlossen werden. Ergebnisse: Durch eine präoperative neurobiologische (Schmerz-)Edukation können postoperative Katastrophisierungstendenzen sowie die postoperative Kinesiophobie positiv beeinflusst werden. Keinen Einfluss hat eine präoperative neurobiologische Edukation auf postoperativen Schmerz und Funktion. Inkonsistenz besteht bislang in der Herangehensweise der PNE (Pain Neuroscience Education).

Schlussfolgerung: Eine präoperative Reduktion von Angst und schmerzaufrechterhaltenden Faktoren v.a. auf psychologischer und sozialer Ebene hat einen positiven Effekt auf die postoperative subjektive Schmerzbewertung, was sich in einer Reduktion von Angst, Katastrophisierungstendenzen und einer geringeren Inanspruchnahme von postoperativen Leistungen im Gesundheitswesen widerspiegelt.

\section{Schlüsselwörter}

Präoperative neurobiologische Edukation · Wirbelsäulenoperation · Angstreduktion · Katastrophisieren · Postoperative Chronifizierung 
Tab. 1 „Key points" der biomedizinischen und neurobiologischen Edukation entsprechend der aktuellen Literatur modifiziert $[13,14,16]$

\begin{tabular}{|l|l|l|}
\hline & Biomedizinische Edukation & Neurobiologische Edukation \\
\hline Hauptpunkte & Erklärung des OP-Prozederes & Physiologie des ZNS und PNS \\
\hline $\begin{array}{l}\text { Herangehens- } \\
\text { weise }\end{array}$ & $\begin{array}{l}\text { Biomedizinische/biomechanische } \\
\text { Herangehensweise }\end{array}$ & Biopsychosoziale Herangehensweise \\
\hline Evidenz & Geringe Evidenz [14] & $\begin{array}{l}\text { Erste evidenzbasierte Ergebnisse weisen } \\
\text { einen guten Effekt auf }\end{array}$ \\
\hline $\begin{array}{l}\text { Schmerz- } \\
\text { spezifizierung }\end{array}$ & $\begin{array}{l}\text { Geringe Schmerzspezifizierung } \\
\text { [13] } \\
\text { Erklärung von (andauerndem) } \\
\text { Schmerz immer als Zeichen einer } \\
\text { Gewebeschädigung [16] }\end{array}$ & $\begin{array}{l}\text { Betrachtung von (andauerndem) } \\
\text { Schmerz unter den Gesichtspunkten } \\
\text { der Plastizität des Nervensystems und } \\
\text { Sensibilisierungsprozesse im ZNS und } \\
\text { PNS. Ziel: Reduktion der Angst vor dem } \\
\text { Schmerz [13, 14, 16] }\end{array}$ \\
\hline $\begin{array}{l}\text { Postulierter } \\
\text { Effekt der } \\
\text { präoperativen } \\
\text { Edukation }\end{array}$ & $\begin{array}{l}\text { Möglicherweise kann sich das } \\
\text { präoperative Angst- und Stress- } \\
\text { level der Patienten steigern, was } \\
\text { sich negativ auf das postoperati- } \\
\text { ve Outcome auswirkt [14] }\end{array}$ & $\begin{array}{l}\text { Reduktion des präoperativen Angst- und } \\
\text { Stresslevels }\end{array}$ \\
\hline ZNS zentrales Nervensystem, PNS peripheres Nervensystem \\
\hline \multicolumn{2}{|l|}{}
\end{tabular}

Trotz erfolgreicher Operation fallen Patienten postoperativ regelmäßig durch kinesiophobes und katastrophisierendes Verhalten sowie anhaltenden Schmerz auf. Es gibt Hinweise, wonach eine ausschließliche biomedizinische Aufklärung (BMA) des OP-Prozederes diese Verhaltensentwicklung begünstigen kann. Im Gegensatz zu einer BMA wird durch eine neurobiologische (Schmerz-)Edukation (PNE) das subjektive Schmerzempfinden vor einem neurobiologischen und neurophysiologischen Hintergrund erklärt. In diesem Review wird der Frage nachgegangen, ob eine zusätzliche perioperative PNE einen positiven Einfluss auf die postoperativen Variablen, insbesondere Kinesiophobie, Katastrophisieren und Schmerz, hat [2].

\section{Einleitung}

Anhaltende Schmerzen sind für den Betroffenen sehr beeinträchtigend und haben häufig negative Auswirkungen auf soziale, familiäre Kontakte und den beruflichen Alltag. Trotz einer sich stetig verbessernden Qualität der OP-Techniken konnte gleichzeitig keine eindeutige physische und mentale postoperative Gesundheitsverbesserung der Patienten erzielt werden [1], was hohe Chronifizierungsraten postoperativer Schmerzen impliziert $[8,13$, 14]. Eine Vielzahl von „randomised controlled trials" (RCT) zeigte bereits einen positiven Effekt durch eine neurobiologische Schmerzedukation („,pain neuro- science education" [PNE]) bei Patienten mit chronischen lumbalen Schmerzen [13, 24]. Diese Überlegungen lassen die Fragestellung $z u$, ob eine präoperative PNE einen positiven Einfluss auf das postoperative Outcome und damit auf geringere postoperative Schmerzchronifizierungsraten hat.

\section{Chronifizierungsgründe}

Nicht selten entwickelt sich eine Schmerzchronifizierung nach einer Operation.

Neuere Untersuchungen von verschiedenen Autoren zeigen, dass bei etwa $40 \%$ aller Patienten, die in den USA aufgrund von degenerativen Veränderungen der Wirbelsäule operiert werden, der Schmerz weit über den normalen Heilungsverlauf einer verletzten Struktur hinaus andauert sie chronifizieren. Zudem weisen Patienten nach einer OP deutliche funktionelle Defizite auf $[1,8,13,14]$.

Die Entwicklung einer (postoperativen) Chronifizierung ist nicht von objektivierbaren Daten (Geschlecht, Familienstand, Ausbildungsstatus) abhängig [7, 30], sondern wird neben einer insuffizienten Stellung der OP-Indikation und einem bereits präoperativ bestehenden chronischen Schmerz insbesondere durch neuroplastische Veränderungen (periphere Sensibilisierung, zentrale Sensibilisierung, deszendierende Modulation) bedingt. Infolgedessen treten häufig maladaptive Copingstrategien, ein Angst-Vermeidungs-
Verhalten, Katastrophisierungsgedanken und negative Emotionen, insbesondere Angst, auf. Schmerzen, welche als bedrohlich empfunden werden, verhindern die Rückkehr zu einer normalen Aktivität [28].

Menschen mit einem ausgeprägten Angst-Vermeidungs-Verhalten (AVV) und Neigungen zur Schmerzkatastrophisierung chronifizieren häufiger $[1,18,20$, 27, 29]. Emotionen und die daraus resultierenden physiologischen Reaktionen eines Menschen können durch Gedanken und Überzeugungen positiv oder negativ beeinflusst werden [27].

Zu den allgemeinen Hauptrisikofaktoren für eine Chronifizierung von Rückenschmerzen gehören der subjektive Umgang mit Arbeitsplatzkonflikten, soziale Isolation, Depressionen, negative Emotionen und Katastrophisierungsgedanken, welche ggf. auch auf eine mangelhafte Aufklärung zurückzuführen sind [7].

\section{Neurobiologische Schmerz- edukation (PNE)}

Neuere Ansätze in der Behandlung chronischer Schmerzsyndrome haben eine neurobiologische Edukation als Haupttherapiebaustein integriert. Ziel dieser Edukation ist es, dem Patienten ein umfassendes Wissen über die neurobiologischen und neurophysiologischen Zusammenhänge des subjektiven Schmerzempfindens zu vermitteln und somit die bedrohende Wirkung des Schmerzes zu reduzieren.

Eine Übersicht der "key points" der biomedizinischen sowie der neurobiologischen Edukation ist in $\mathbf{0}$ Tab. 1 beschrieben, generelle Eigenschaften bezüglich der Inhalte einer PNE finden sich in - Tab. 2.

In einem RCT von Louw et al. (2014) konnte bereits ein positiver Effekt in Bezug auf den Schmerz, das Katastrophisieren und die Funktion/Beweglichkeit durch eine PNE bei Patienten mit chronischen lumbalen Schmerzen im 1-Jahres-Followup zeigen $[13,24]$. Schmerz als solcher hat primär die Funktion der "Warnung vor einer Bedrohung" und stellt somit eine Schutzfunktion vor einer potenziellen Gefahr dar. Allein das Verstehen des Schmerzes führt zu einer Reduktion der subjektiv empfundenen Bedrohung durch den Schmerz [3, 
Tab. 2 Leitfaden nach Louw (2012) für die neurobiologische Edukation vor einer Wirbelsäulenoperation [12]

Inhaltspunkte der PNE

1. Bestärkung der Entscheidung zu einer Operation

2. Anatomie und Physiologie des Nervensystems

3. Das Nervensystem und der Rücken

4. Das Nervensystem im Kontext einer OP

5. Schmerzregulierung im zentralen Nervensystem

6. Genesung nach der Operation

PNE Pain Neuroscience Education

8]. Diese Überlegungen lassen die Fragestellung zu, welchen Effekt eine präoperative PNE auf das postoperative Outcome hätte.

Das Ziel dieses systematischen Reviews ist, den Effekt einer präoperativen neurophysiologischen Schmerzedukation auf das postoperative Outcome sowie die unterschiedlichen Herangehensweisen (bzgl. Dauer und Setting) dieser Intervention zu analysieren.

Die primäre Forschungsfrage hierzu lautet: Welchen Effekt zeigt eine präoperative neurophysiologische Schmerzedukation auf das postoperative Outcome?

Die sekundäre Forschungsfrage lautet: Welche Herangehensweisen wählen die Autoren zur präoperativen neurophysiologischen Schmerzedukation?

\section{Methode}

Die medizinische Datenbank PubMed wurde nach englischen und deutschen Artikeln durchsucht. Bei der Suche wurde nach dem $\mathrm{PI}(\mathrm{C})$ O-Prinzip vorgegangen und die Suchbegriffe aus Tab. 3 verwendet. Da die einfache Suche in „All Fields" sehr unspezifisch war und sehr hohe Trefferzahlen zur Folge hatte, wurde anschließend mit der MeSH(Medical Subject Headings)Term-Funktion und in der Kategorie "Title/ Abstract" gesucht.

\begin{tabular}{|l|l|l|}
\hline Tab. 3 Suchbegriffe, Funktion und Trefferzahl \\
\hline Suchbegriffe & Funktion & Treffer \\
\hline Chronic pain & MeSH Terms & 14.774 \\
\hline Low back pain & MeSH Terms & 21.965 \\
\hline Pain neuroscience education & MeSH Terms & 248 \\
\hline Preoperative pain neuroscience education & MeSH Terms & 12 \\
\hline Pain & Title/Abstract & 631.164 \\
\hline Disability & Title/Abstract & 158.684 \\
\hline Kinesiophobia & Title/Abstract & 873 \\
\hline Catastrophizing & Title/Abstract & 3197 \\
\hline Central sensitization & Title/Abstract & 2876 \\
\hline Central sensitization & MeSH Terms & 634 \\
\hline MeSH Medical Subject Headings & & \\
\hline
\end{tabular}

Schritte der systematischen Literaturrecherche

Die Verknüpfung wurde durch die Boulschen Operatoren "AND" und "OR" wie folgt vorgenommen:

\section{Verknüpfung. - 75 Treffer:}

(()(((chronic pain[MeSH Terms]) AND (pain neuroscience education[MeSH Terms])) AND (pain[Title/Abstract])) OR (disability[Title/Abstract])) AND (kinesiophobia[Title/Abstract])) OR (catastrophizing[Title/Abstract])) AND (central sensitization[Title/Abstract])

\section{Verknüpfung. - 2 Treffer:}

(((chronic pain[MeSH Terms]) AND (pain neuroscience education[MeSH Terms])) AND (pain[MeSH Terms])) AND (central sensitization[MeSH Terms])

\section{Verknüpfung. - 6 Treffer:}

(()(((low back pain[MeSH Terms]) AND (preoperative pain neuroscience education)) OR (preoperative management)) AND (pain[Title/Abstract])) OR (disability[Title/Abstract])) AND (kinesiophobia[Title/Abstract])) AND (catastrophizing[Title/Abstract])) AND (central sensitization[Title/Abstract])

Auf Basis der Abstracts wurden 83 Artikel entsprechend den unten aufgeführten Ein- und Ausschlusskriterien gescreent.

Zur Vertiefung der Thematik wurde bei google.scholar.com und scinos.de nach Artikeln gesucht. Auch wurden Verweise in den vorliegenden Artikeln und Studien auf die dort verwendeten Quellenangaben aufgenommen.

\section{Einschlusskriterien}

Die Artikel wurden eingeschlossen, wenn sie ab dem 01.01.2011 erschienen sind, ein Evidenzlevel 1, 2, 3 oder 4 erfüllten, eine biomedizinische und/oder eine neurobiologische Edukation bei Patienten vor einer Operation durchgeführt wurde und $z u$ den untersuchten Outcomeparametern die medizinisch entstehenden Kosten, Schmerz, Funktion, Katastrophisierung und/oder Kinesiophobie zählten.

Ausgeschlossen wurden die Artikel bei einem Erscheinungsdatum vor 01.01.2011, mit einem Evidenzlevel 5, wenn keine präoperative Edukation durchgeführt wurde sowie bei anderen als den oben erwähnten Outcomeparametern.

\section{Ergebnisse}

Nach dem Screening der Abstracts wurden neun Studien $[10,11,13-16,19,25,26]$ eingeschlossen, welche an $n=360$ Probanden den zusätzlichen Effekt von verschiedenen präoperativen Edukationen auf das postoperative Outcome untersuchten. Der Fokus wurde hierbei auf sieben Forschergruppen [11, 13-16, 19, 25] gelegt, welche an insgesamt $n=213$ Patienten den zusätzlichen Effekt einer präoperativen PNE zu einer biomedizinischen Edukation $(n=114)$ im Vergleich zu einer alleinigen präoperativen biomedizinischen Edukation $(n=99)$ bei Patienten vor Operationen an verschiedenen Körperbereichen untersuchten. Zwei Untersuchungen [10, 26] verglichen an insgesamt 147 Patienten vor einer Wirbelsäulenoperation weitere Formen präoperativer Aufklärung 


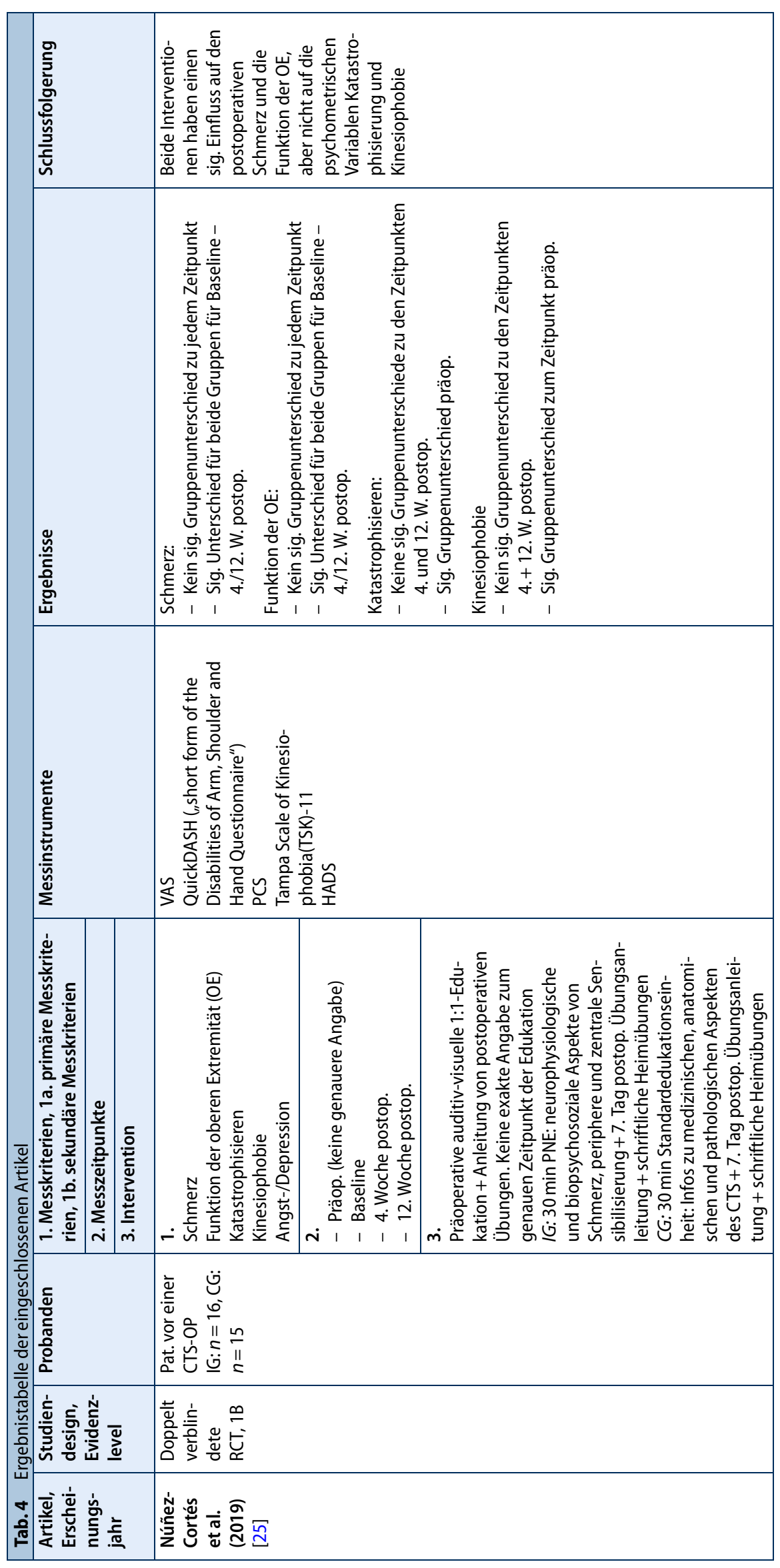




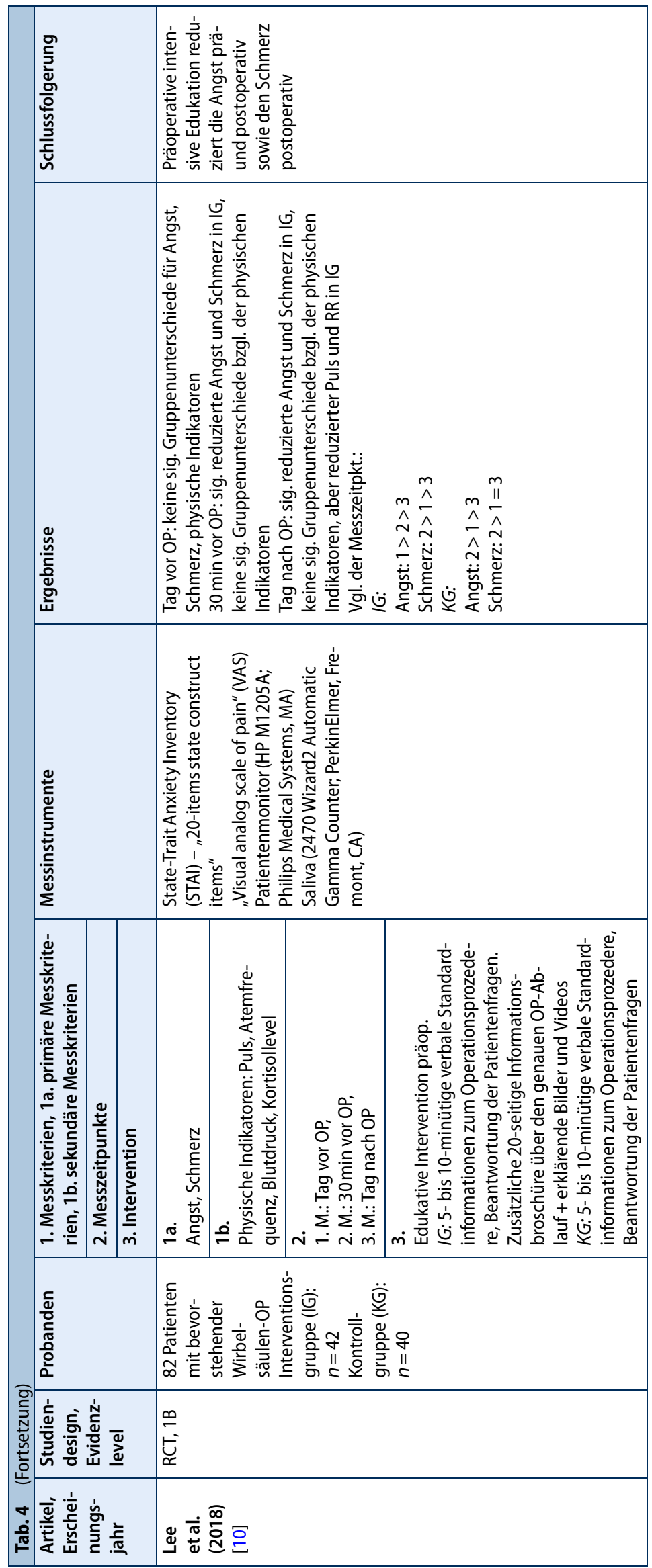

(Führung durch die Klinikräumlichkeiten, zusätzlich schriftliches Material über das OP-Prozedere, detailliertere Erklärungen des OP-Prozederes etc.).

Sechs der eingeschlossenen Studien weisen ein sehr gutes Evidenzlevel (1 bzw. 1B) auf [10, 11, 13, 16, 25, 26]. Drei Studien haben eine geringere Evidenz (3B und $4 ;[14,15,19])$.

Aus den eingeschlossenen Studien geht hervor, dass bislang kein standardisiertes Vorgehen einer präoperativen PNE existiert. Ein Großteil der Autoren bezieht sich auf das Vorgehen der Arbeitsgruppen von Louw et al. Nahezu keine Angaben machen die Autoren für den genauen Zeitpunkt der präoperativen PNE. Lediglich von einer Forschungsgruppe wird angegeben, dass die edukativen Einheiten in den Wochen 8, 7,6 und 5 vor der OP stattfinden sollen [11]. Louw et al. [14] führen die präoperative PNE 28 bis 2 Tage vor der OP durch. Nach Louw et al. [13] ist der beste Zeitpunkt einer präoperativen Edukation eine Woche vor der OP.

Eine größere Konsistenz besteht zwischen den Autoren für die Art der Informationsvermittlung und das Setting.

Alle Autoren, welche eine PNE durchführten, wählten ein auditiv-visuelles Vorgehen [11, 13-15, 19, 25]. Außer von Núñez-Cortés et al. [25] wurde dieses durch schriftliches Material untermauert. Das gewählte Setting war bei allen Untersuchungen, welche die positiven Aspekte einer Kleingruppe in den Vordergrund stellten, im 1:1-Kontakt.

Weniger Konsistenz besteht für die Dauer und Anzahl der präoperativen PNE, wobei ein Großteil aller Autoren eine Einheit von 30 min durchführte [13-15, 19, 25]. Eine Forschungsgruppe führte vier Einheiten mit einer Dauer von 20 bis $60 \mathrm{~min}$ im Abstand von einer Woche durch [11]. Lediglich bei einer Erhebung wurde eine präoperative PNE mit einer postoperativen Eigenübungstherapie kombiniert [25] und eine weitere Untersuchung kombinierte eine präoperative Edukation mit präoperativer Mobilisation [11].

Im Durchschnitt erhielten die Patienten eine präoperative PNE-Einheit von $35 \mathrm{~min}$.

Zusammenfassend herrscht Uneinigkeit hinsichtlich der Art, Dauer und des Settings. Überwiegend wurde eine einmalige 30-minütige visuell-auditive Edukati- 


\begin{tabular}{|c|c|c|c|c|c|c|}
\hline \multirow[t]{2}{*}{ 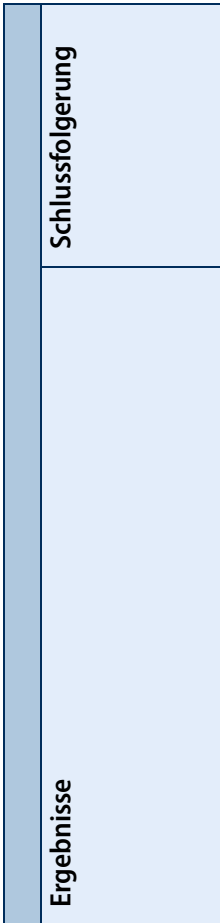 } & \multicolumn{6}{|c|}{ 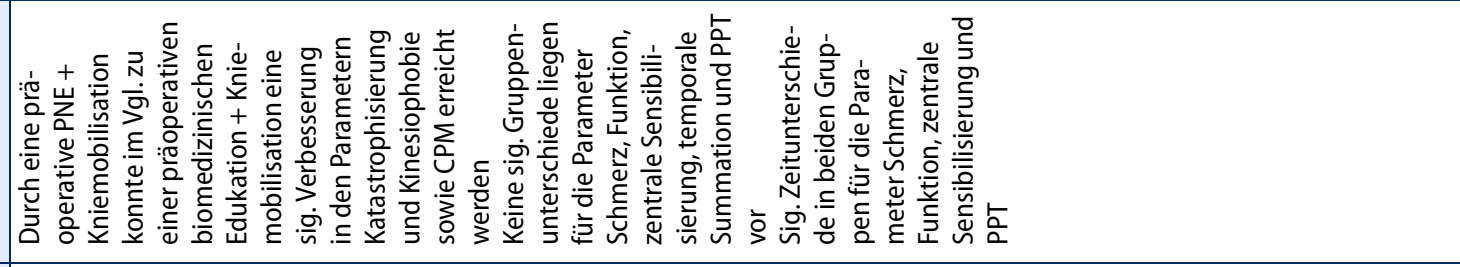 } \\
\hline & 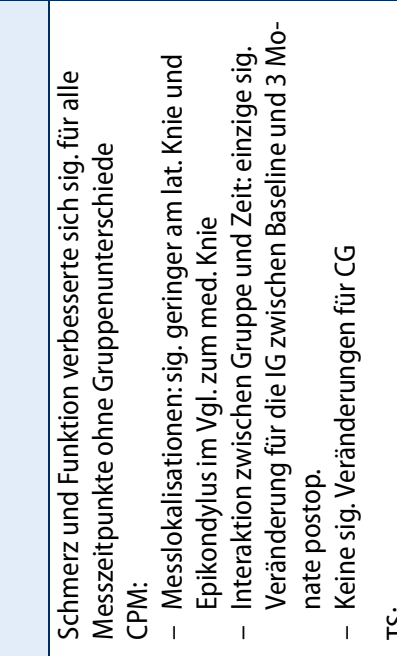 & 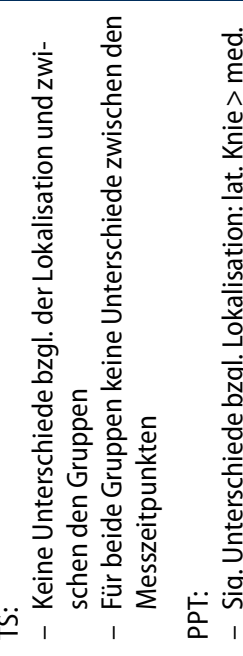 & 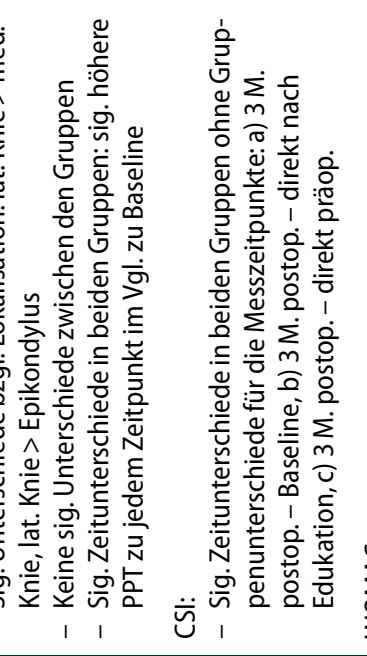 & 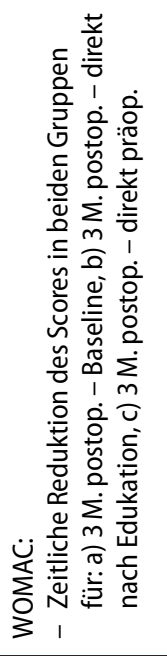 & 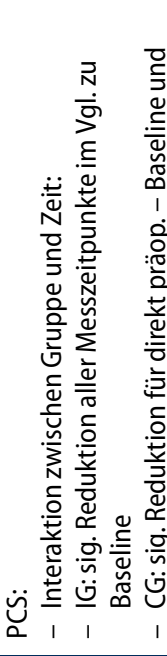 & 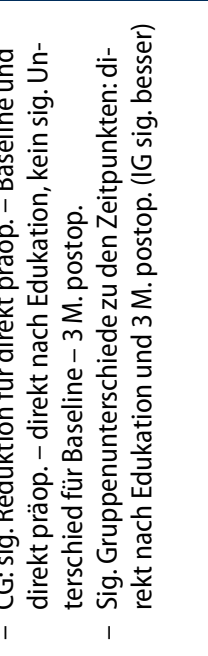 \\
\hline 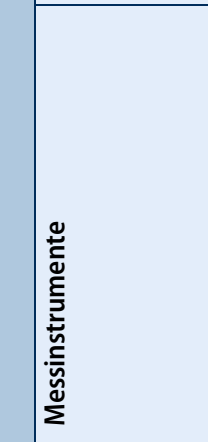 & \multicolumn{6}{|c|}{ 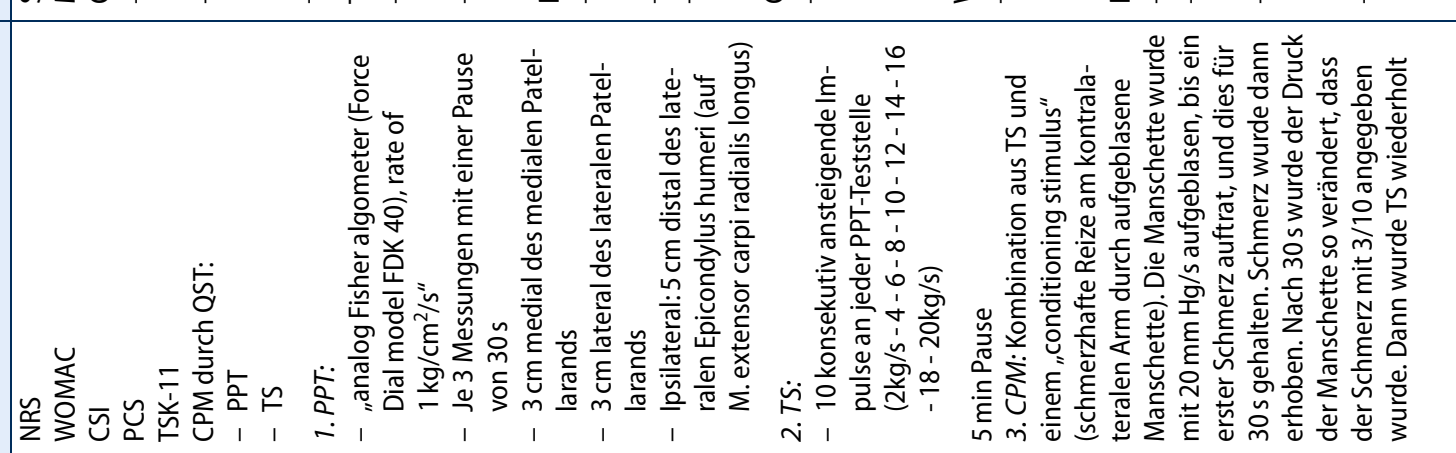 } \\
\hline 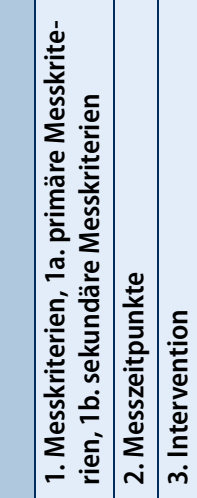 & 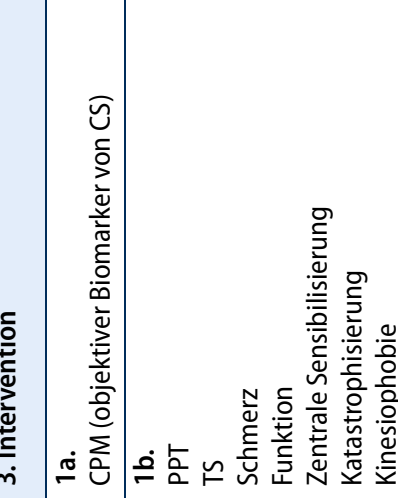 & 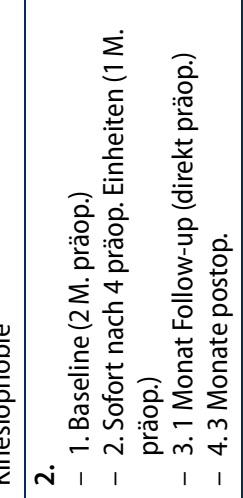 & 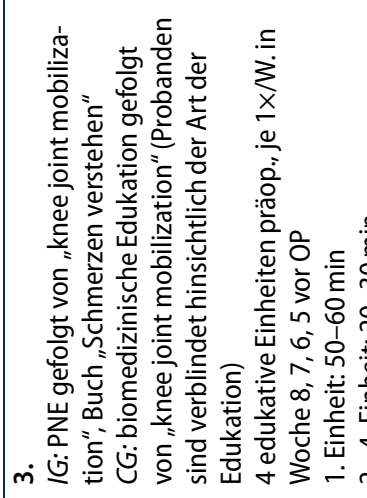 & 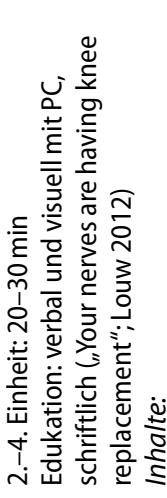 & 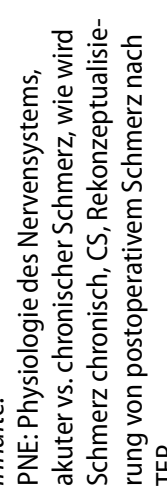 & 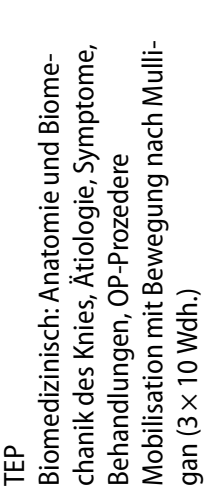 \\
\hline 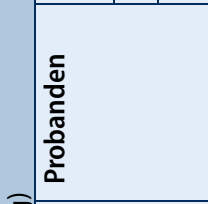 & \multicolumn{6}{|l|}{ 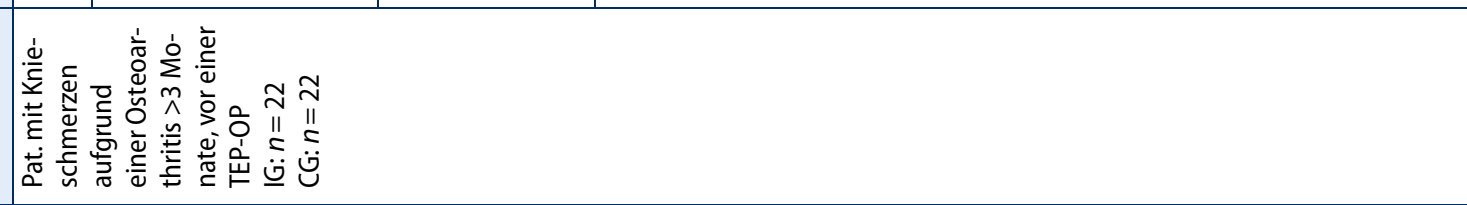 } \\
\hline 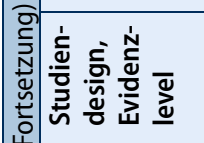 & \multicolumn{6}{|l|}{ 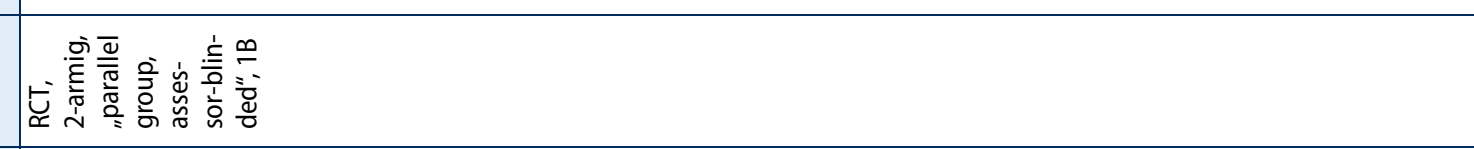 } \\
\hline 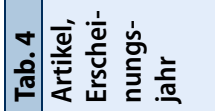 & \multicolumn{6}{|l|}{ 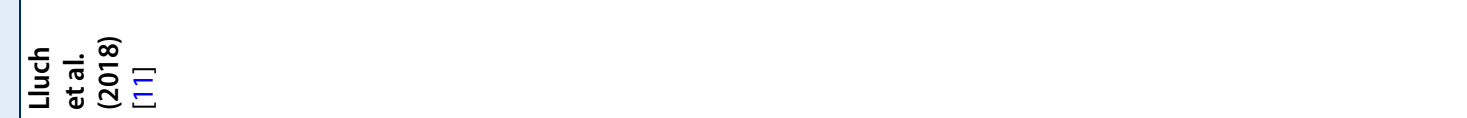 } \\
\hline
\end{tabular}




\begin{tabular}{|c|c|c|c|c|}
\hline 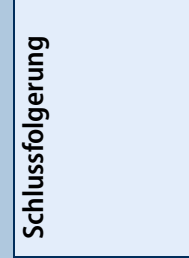 & & \multicolumn{3}{|c|}{ 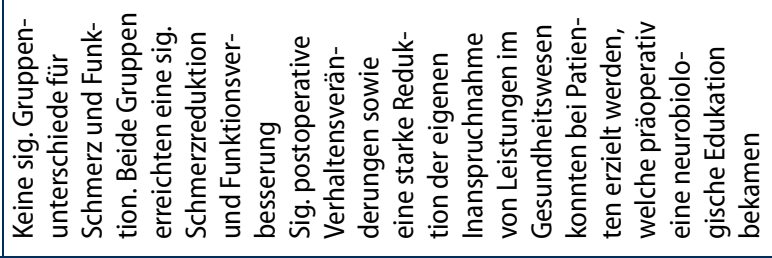 } \\
\hline 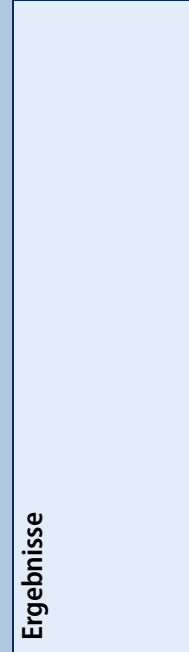 & 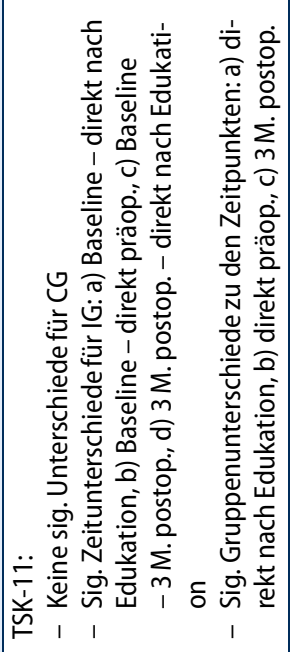 & \multicolumn{3}{|c|}{ 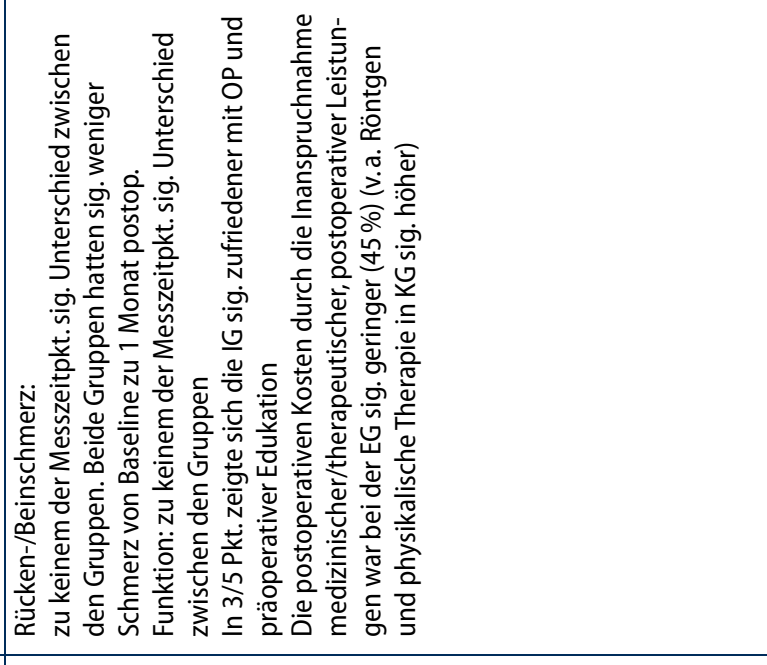 } \\
\hline 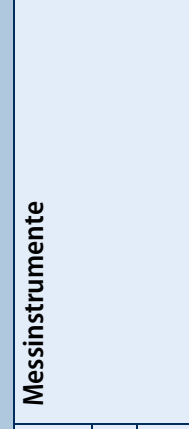 & & \multicolumn{3}{|c|}{ 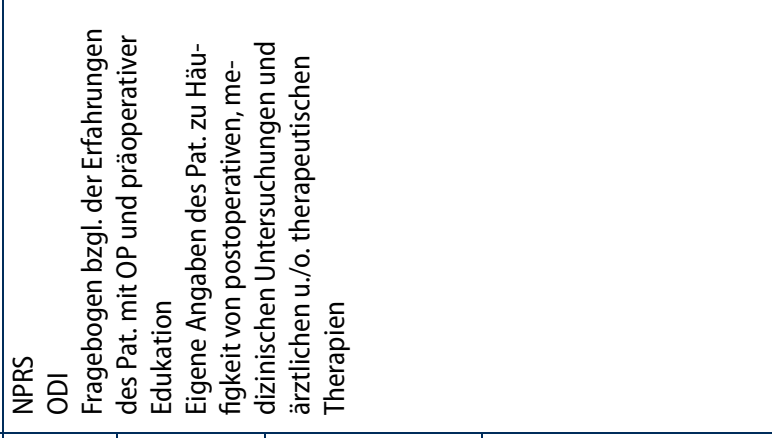 } \\
\hline 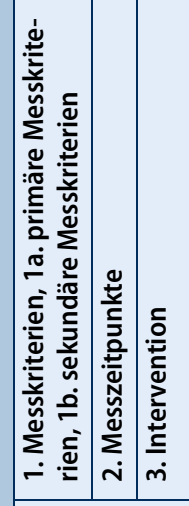 & & 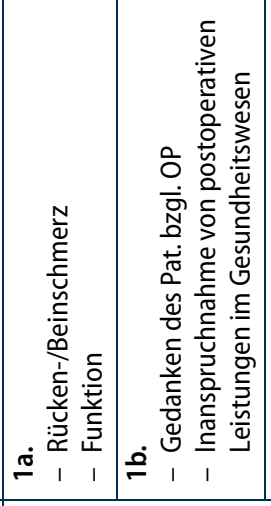 & 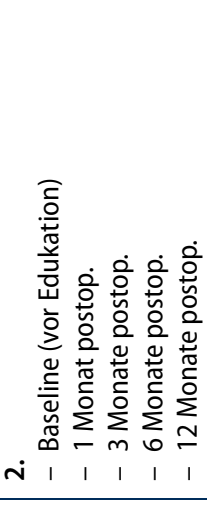 & 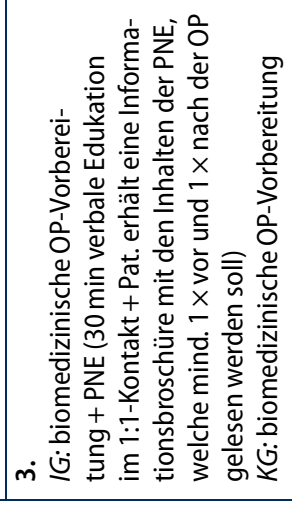 \\
\hline 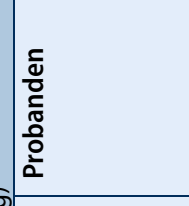 & & 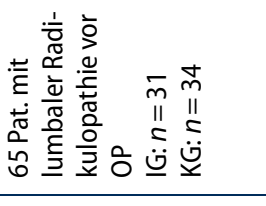 & & \\
\hline 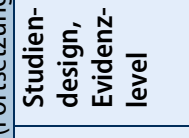 & & 竧竧密总 & & \\
\hline 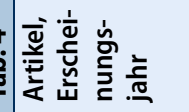 & & 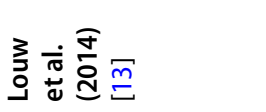 & & \\
\hline
\end{tabular}




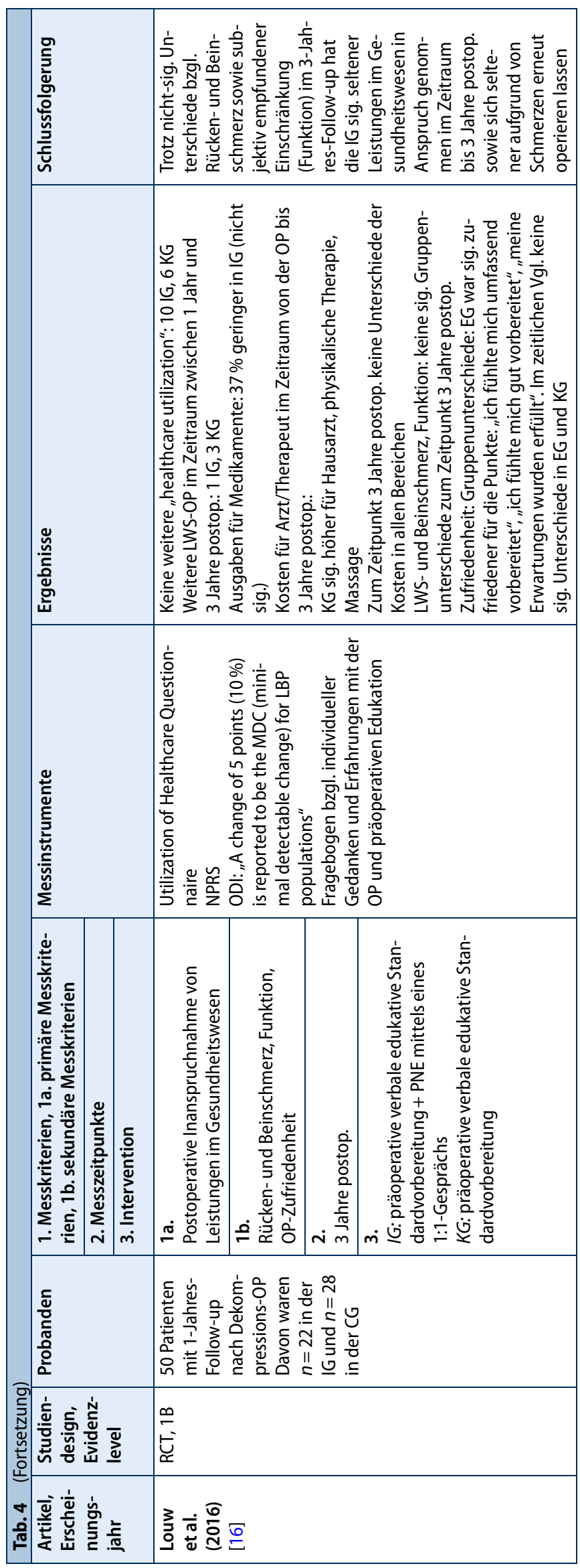

on mit zusätzlichem schriftlichem Material im 1:1-Kontakt durchgeführt. Über den Zeitpunkt der Edukation kann aufgrund der großen Inkonsistenz keine Aussage getroffen werden. Von den meisten Autoren wurde ein zeitlicher Abstand zwischen der Edukation und der OP von mehr als einem Tag gewählt.

Die Autoren untersuchten v.a. die Domänen Schmerz, Funktionseinschränkung, Katastrophisierung, Kinesiophobie sowie die postoperative Inanspruchnahme von Leistungen im Gesundheitswesen.

Die Ergebnisse zeigen Tendenzen auf, wonach eine präoperative PNE v.a. die psychometrischen Variablen Katastrophisierung (kein Effekt $(n=43)[19,25]$; signifikanter Effekt $(n=55)[11,14,15])$. Kinesiophobie (kein Effekt [25]; signifikanter Effekt $[11,19])$ sowie die Sensitivität des Nervensystems im betroffenen Körpergebiet (kein Effekt [11]; signifikanter Effekt $[15,19])$ positiv beeinflusst. Keinen signifikanten Effekt hat eine präoperative PNE auf den postoperativen Schmerz und Funktion. Eine Intensivierung der biomedizinischen Aufklärung kann die postoperative Angst steigern [26]. Wie die Follow-upErhebungen zeigten $[13,16]$, scheint eine postoperative Verhaltensveränderung im Sinne einer signifikant geringeren erneuten Inanspruchnahme von Leistungen im Gesundheitswesen nicht in Zusammenhang mit Schmerz und Funktion zu stehen, sondern eher mit den psychometrischen Variablen Katastrophisierung und Bewegungsangst.

Eine Übersicht der eingeschlossenen Artikel gibt - Tab. 4 mit den entsprechenden Abkürzungen in $\bullet$ Tab. 5.

\section{Diskussion}

Für die Überprüfung der Validität der Primärstudien spielt die Untersuchung eines möglichen Bias eine wichtige Rolle. Wichtige Parameter sind in diesem Zusammenhang die untersuchte Population sowie Verblindungs- und Randomisierungsprozesse. Die Validität der Primärstudien wird überwiegend als gut bezeichnet (gute Validität [10, 11, 13, 16, 25]; mäßige Validität [19]; geringe Validität [14, 15]). Die Generalisierbarkeit der Ergebnisse auf weitere Populationen ist eher gering (hohe Generalisierbarkeit [10]; geringe Generalisier- 


\begin{tabular}{|c|c|c|c|c|}
\hline 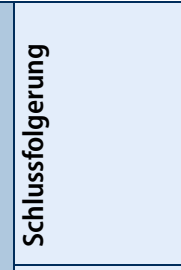 & \multicolumn{4}{|c|}{ 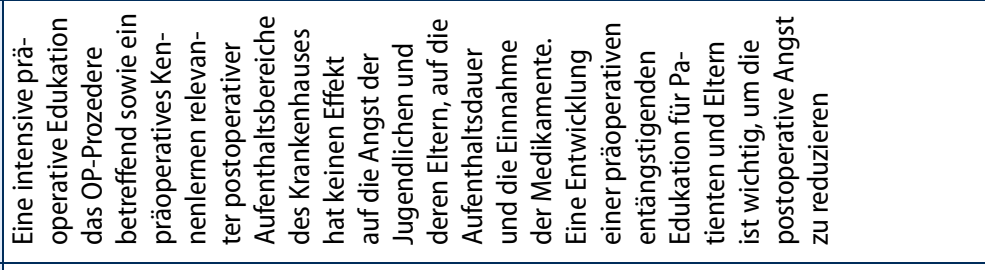 } \\
\hline 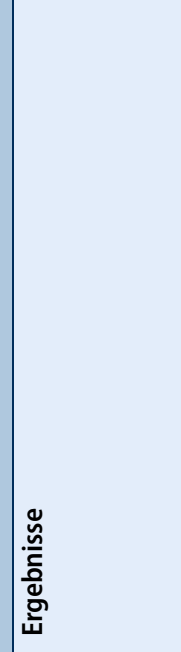 & \multicolumn{4}{|c|}{ 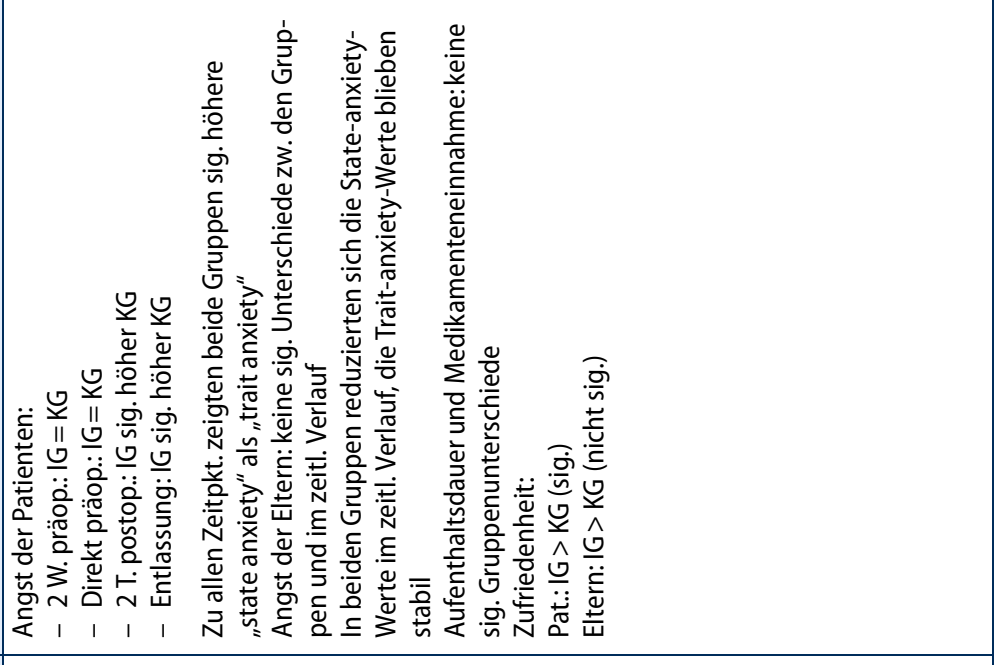 } \\
\hline 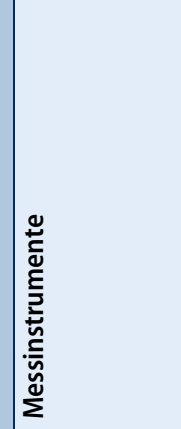 & \multicolumn{4}{|l|}{ 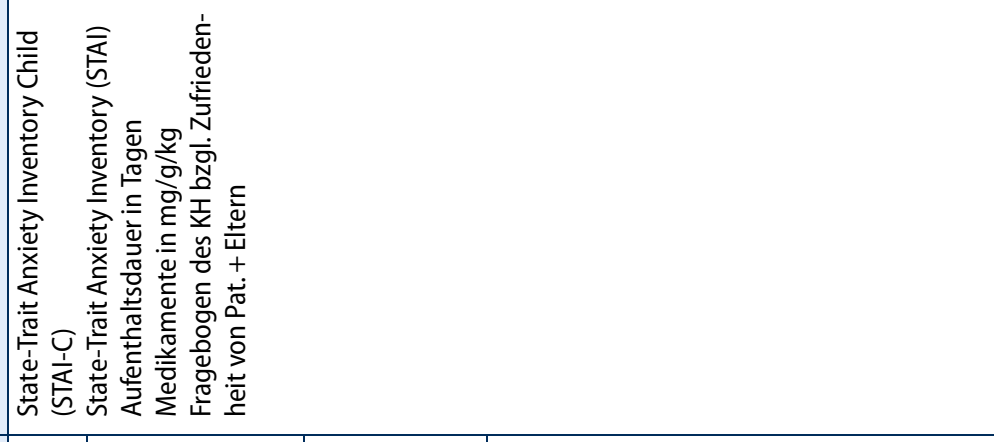 } \\
\hline 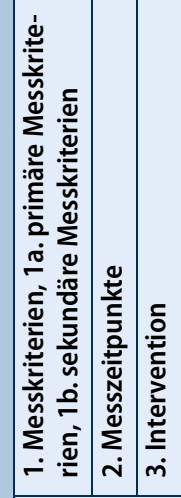 & 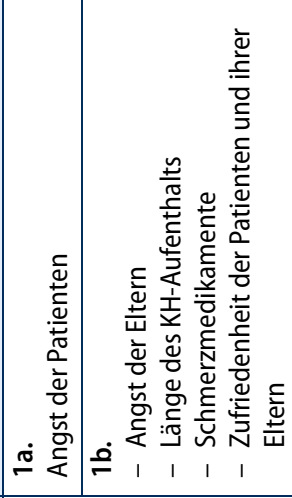 & 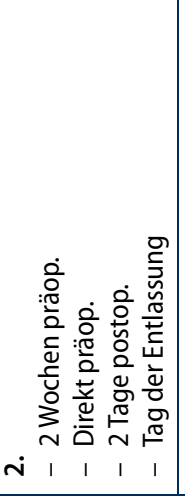 & 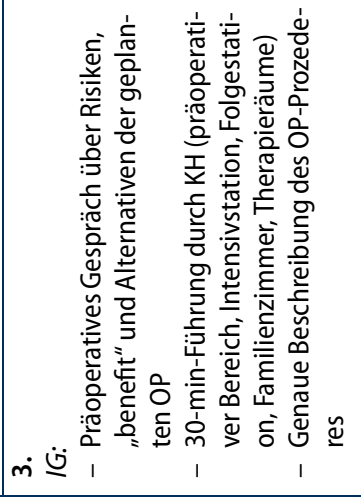 & 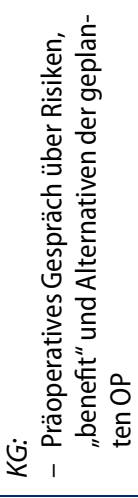 \\
\hline 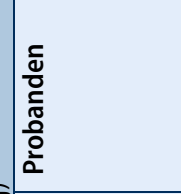 & \multicolumn{4}{|l|}{ 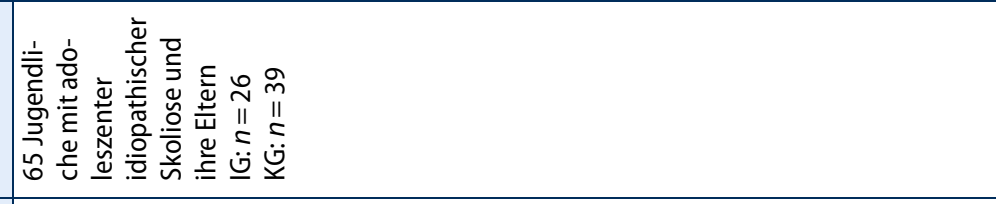 } \\
\hline 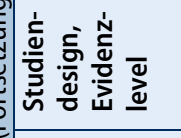 & \multicolumn{4}{|l|}{ 离 } \\
\hline 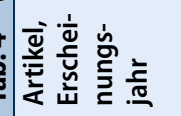 & \multicolumn{4}{|l|}{ 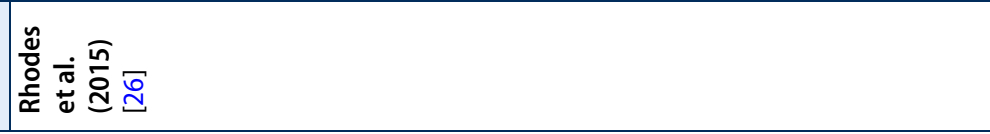 } \\
\hline
\end{tabular}




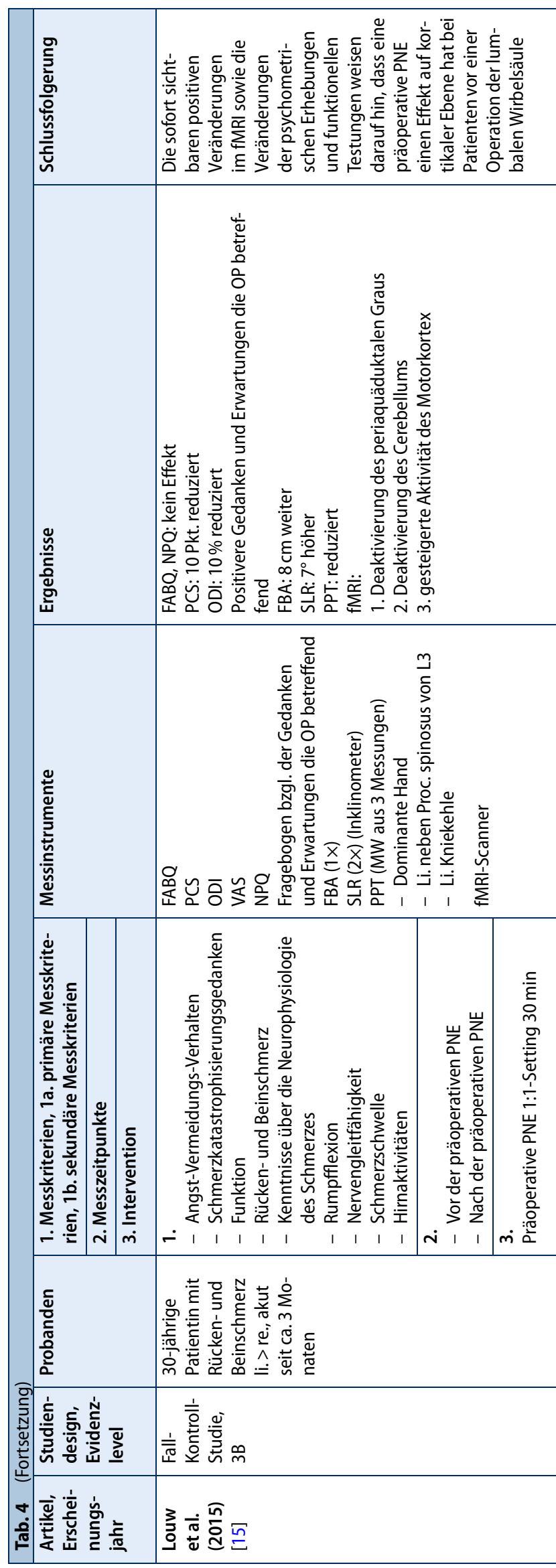

barkeit $[11,13-16,19,25,26])$. Kritisch zu betrachten sind die Ergebnisse v.a. deshalb, da es sich in einigen Studien um sehr kleine Populationsgruppen handelt bzw. z. T. eine Kontrollgruppe fehlt.

In Bezug auf die primäre Forschungsfrage besteht der Wert der Studien v.a. darin, dass Tendenzen aufgezeigt werden konnten, welche eine positive Beeinflussung des postoperativen Outcomes durch eine edukative Intervention vor einer Operation belegen. Diese zeichnen sich v.a. durch eine Veränderung der psychometrischen Variablen (Kinesiophobie und Katastrophisierung) sowie durch eine reduzierte Sensitivität des Nervensystems aus. Keine Veränderungen zeigten sich hinsichtlich Schmerzintensität und Funktion. Dass trotzdem eine Verhaltensveränderung im Sinne einer signifikant geringeren Inanspruchnahme von postoperativen Leistungen im Gesundheitswesen zu beobachten ist, zeigt eindeutig, dass Patienten nicht aufgrund der alleinigen Schmerzintensität oder der eingeschränkten Funktion nach externer Hilfe suchen, sondern auch aufgrund der damit verbundenen Ängste und Sorgen, welche u. a. durch Schmerzen ausgelöst werden können. Kritisch anzumerken ist an dieser Stelle, dass von den meisten Autoren diese Ergebnisse nur maximal in einem 3-Monats-Follow-up bestätigt wurden, über langfristigere Outcomes sind bislang nur wenige Ergebnisse bekannt $[13,14,16]$. Ein 12- und 24-MonatsFollow-up ist in dem Studienprotokoll von Ickmans et al. (2016) vorgesehen [8], welches aufgrund der Einschlusskriterien nicht in die Ergebnisauswertung aufgenommen wurde.

Bezüglich der sekundären Forschungsfrage zeigen die Studien eine größere Inkonsistenz in der Herangehensweise der präoperativen PNE. Zwar überschneiden sich größtenteils die Inhalte der präoperativen $\mathrm{PNE}$, allerdings liegen Variationen in Zeitpunkt und Dauer vor. Einigkeit herrscht hinsichtlich eines visuell-auditiven Settings mit unterstützendem schriftlichen Material. Welchen Umfang das schriftliche Material hat, ob hierbei viele Bilder oder Verlinkungen zu weiteren Informationsquellen geboten werden, bleibt meist unklar. Auch wurde die präoperative Edukation überwiegend im 1:1-Kontakt durchgeführt. Die positiven Aspekte einer 


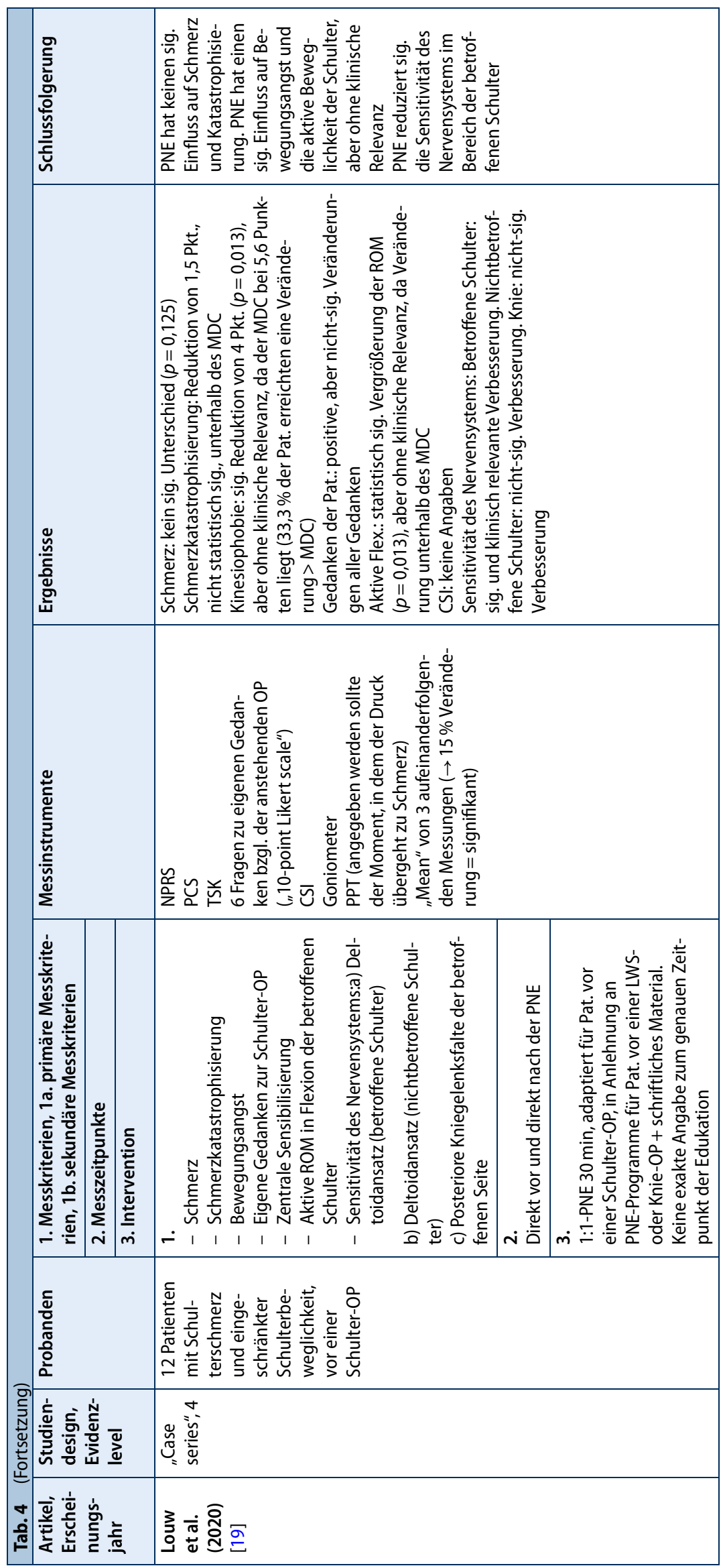

Gruppentherapie, welche im nichtoperativen Setting zu guten Ergebnissen führten, wurden von den Autoren nicht beleuchtet. Bislang gibt es keine Untersuchungen zu den Effekten einer präoperativen PNE im Gruppensetting. Ob der von den Forschern gewählten Herangehensweise im 1:1-Kontakt ein übergeordneter Effekt zugeschrieben werden kann, kann nicht beantwortet werden.

Obwohl in dem bereits in 2. Auflage erschienenen Leitfaden „Pain Neuroscience Education" von Louw et al. (2018) [17] eindeutig eine Kombination aus PNE und Übungstherapie empfohlen wird, führten lediglich zwei Autoren [11, 25] eine solche kombinierte Herangehensweise durch. Auch hier liegen somit Unstimmigkeiten in der Herangehensweise vor.

Zusammenfassend sind die ausgewählten Primärstudien v.a. für die Beantwortung der primären Forschungsfrage von großer Bedeutung. Sie zeigen einheitlich die Tendenz auf, dass eine präoperative PNE zu einer Verbesserung psychometrischer Variablen führt und somit das postoperative Outcome nicht durch die Parameter "Schmerz" und "Funktion" bestimmt wird. In Bezug auf die sekundäre Forschungsfrage legen sie in der gemeinsamen Betrachtung Unklarheiten hinsichtlich einer evidenzbasierten Herangehensweise offen.

Ein möglicher Bias kann gegebenenfalls dadurch entstehen, dass fünf der neun eingeschlossenen Studien von Louw et al. durchgeführt wurden.

Mögliche neurobiologische Mechanismen der positiven Effekte von PNE

Eine Vielzahl von kortikalen Regionen, welche für das Verhalten, Emotionen, Erfahrungen etc. verantwortlich sind, ist während einer Schmerzwahrnehmung gleichzeitig aktiv und durch Nervenzellen miteinander verknüpft. Dieses sich permanent verändernde Netzwerk von Nervenzellen wird Schmerzneuromatrix oder Konnektom genannt $[9,23,30]$. Dysfunktionen im zentralen und/oder peripheren Nervensystem unterhalten die Aktivitäten in der kortikalen Matrix und können zu nicht zielführenden Copingstrategien führen [5]. 


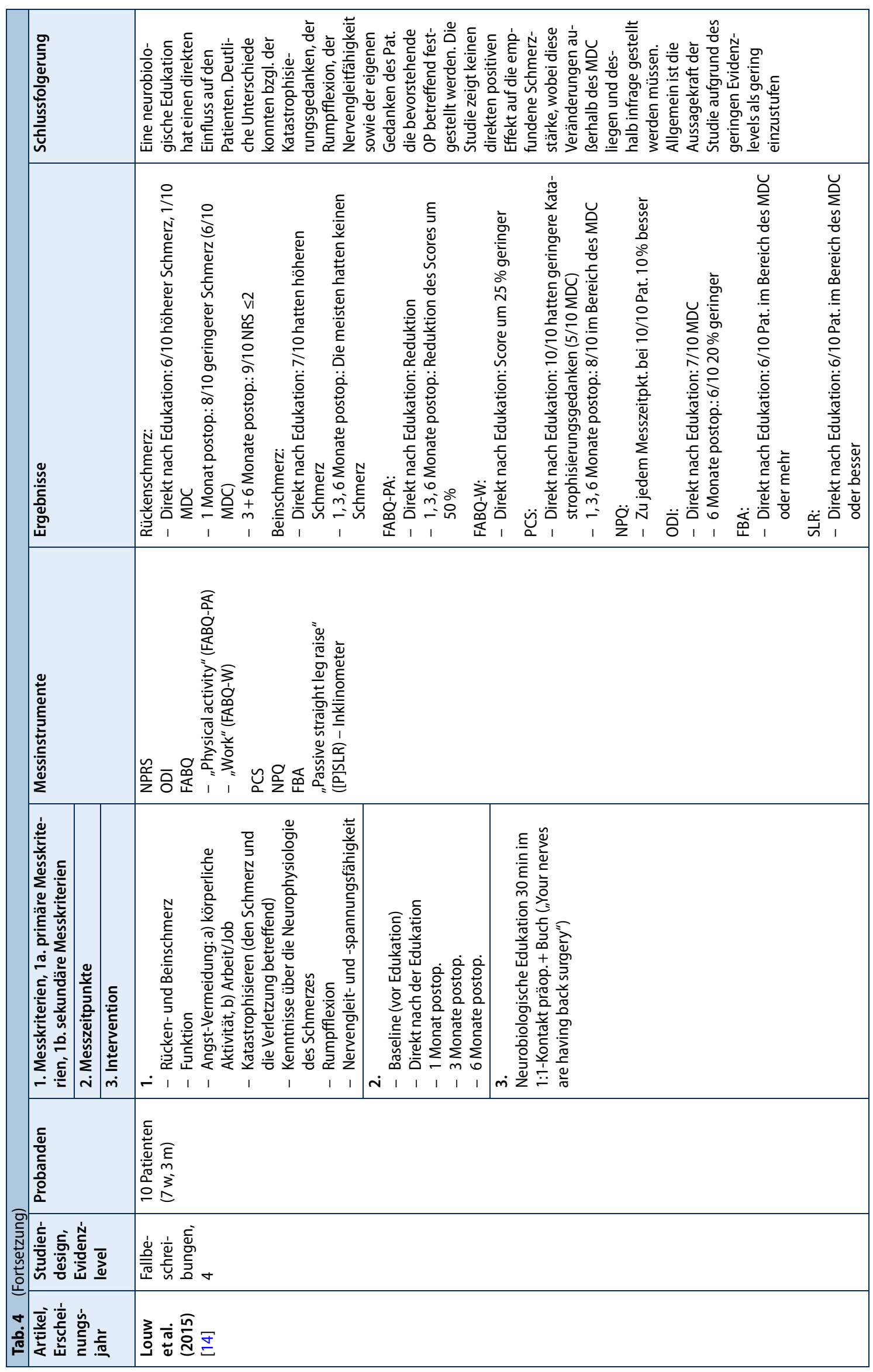




\begin{tabular}{|c|c|}
\hline CPM & "Conditioned pain modulation" \\
\hline CSI & "Central sensitization inventory" \\
\hline CTS & Karpaltunnelsyndrom \\
\hline DASH & $\begin{array}{l}\text { Disability of Arm, Shoulder and } \\
\text { Hand Questionnaire }\end{array}$ \\
\hline FABQ & $\begin{array}{l}\text { Fear Avoidance Belief Question- } \\
\text { naire }\end{array}$ \\
\hline FBA & Finger-Boden-Abstand \\
\hline HADS & $\begin{array}{l}\text { Hospital Anxiety and Disability } \\
\text { Questionnaire }\end{array}$ \\
\hline IG & Interventionsgruppe \\
\hline KG & Kontrollgruppe \\
\hline LBP & "Low back pain" \\
\hline MDC & "Minimal detectable change" \\
\hline NPQ & $\begin{array}{l}\text { Neurophysiology of Pain Ques- } \\
\text { tionnaire }\end{array}$ \\
\hline NPRS & "Numeric pain rating scale" \\
\hline NRS & "Numeric rating scale" \\
\hline ODI & Oswestry Disability Index \\
\hline $\mathrm{OE}$ & Obere Extremität \\
\hline PCS & "Pain catastrophizing scale" \\
\hline PNE & „Pain neuroscience education“ \\
\hline PPT & „Pain pressure threshold" \\
\hline QST & Quantitative sensorische Testung \\
\hline SLR & "Straight leg raise" \\
\hline STAI & State-Trait Anxiety Inventory \\
\hline TEP & Totalendoprothese \\
\hline TS & Temporale Summation \\
\hline TSK & Tampa Scale of Kinesiophobia \\
\hline VAS & Visuelle Analogskala \\
\hline WOMAC & $\begin{array}{l}\text { Western Ontario and McMaster } \\
\text { Universities Osteoarthritis Index }\end{array}$ \\
\hline \multicolumn{2}{|c|}{$\begin{array}{l}\text { RCT Randomized Controlled Trial, CG Control } \\
\text { group, IG Interventriosngruppe, } f M R T \text { funktio- } \\
\text { nelle Magnetresonanztomografie }\end{array}$} \\
\hline
\end{tabular}

Die fMRT-Untersuchungen von Louw et al. [15] zeigten nach der PNE eine Deaktivierung in Arealen des periaquäduktalen Graus (PAG) und des Cerebellums sowie eine gesteigerte Aktivität im Motorkortex. Besonders dem PAG und dem Cerebellum wird eine wichtige Rolle in der absteigenden Schmerzmodulation, dem Vermeidungsverhalten sowie dem subjektiven Empfinden einer Bedrohung zugeschrieben. Das PAG steht über direkte Bahnen mit dem Gyrus cinguli in Verbindung, welcher an der emotionalen Bewertung von Schmerz beteiligt ist $[1,4]$. Wie lange die gefundenen kortikalen Veränderungen bei der untersuchten Patientin bestehen blieben, ist aufgrund fehlender weiterer
Messungen unklar. Wichtig erscheint, dass diese Ergebnisse aufgrund des Einzelfalldesigns nicht generalisierbar sind.

Die durch eine Schmerzerfahrung hervorgerufenen kortikalen neurobiologischen Veränderungen im Sinne einer zentralen Sensibilisierung stehen in einem engen Zusammenhang mit den psychometrischen Charakteristika Kinesiophobie und Katastrophisieren [22]. Diese enge Verknüpfung von Schmerz und Emotionen wird auch in einem Review von Lumley et al. (2011; [21]) beleuchtet.

Um die Entwicklung kinesiophober und katastrophisierender Verhaltensweisen zu verhindern oder deren weitere Ausprägung zu reduzieren, ist es fundamental, den Schmerz sowohl bei akuten als auch bei chronischen Schmerzsyndromen für seine Bewältigung zu verstehen [3, 5].

Die Rekonzeptualisierung von Schmerz und einer Schmerzerfahrung steht im Hauptfokus der PNE.

Zahlreiche Autoren vertreten die Meinung, dass das postoperative Outcome bereits durch die präoperative Phase mit beeinflusst werden kann [11, 13-15, 19].

Auf Basis der Ergebnisübersicht kann hypothetisiert werden, dass eine präoperative neurobiologische Erklärung von Schmerz im postoperativen Stadium die Sensitivität des Nervensystems sowie kinesiophobe und schmerzkatastrophisierende Tendenzen reduziert. Diese Ergebnisse konnten unabhängig von der Lokalisation des OP-Bereichs bestätigt werden.

Durch Langzeit-Follow-up-Erhebungen konnte eine Verhaltensveränderung im Sinne einer geringeren Inanspruchnahme von postoperativen Leistungen im Gesundheitswesen beobachtet werden.

Keinen Einfluss hat eine präoperative PNE auf die postoperative Schmerzintensität und Funktion.

Hinsichtlich der Herangehensweisen liegen v.a. in Zeitpunkt und Dauer der präoperativen PNE Inkonsistenzen vor. Um den Effekt der präoperativen PNE zu stärken sowie ihren Nutzen weiter zu optimieren, besteht hier weiterer Forschungsbedarf.

Die Analyse der Herangehensweise von PNE (sowohl bei akuten und chronischen Schmerzen als auch im präoperativen Kontakt) im klinischen Setting offenbart die Notwendigkeit einer intensiven Erklä- rung von Schmerz. Dass der empfundene Schmerz immer eine Leistung des Gehirns ist, sollte unabhängig von körperlicher Diagnose und Heilungsstadium der Struktur immer Teil eines aufklärenden Gesprächs aller Berufsgruppen sein. Die Erläuterung einer Funktionsstörung, welche alleinig für einen Schmerz verantwortlich gemacht wird, reicht für keinen Schmerzphänotyp aus, um die Bedrohung eines Schmerzes zu reduzieren.

Kritisch ist zu betrachten, dass die durchgeführten präoperativen PNE-Interventionen ausschließlich im 1:1-Setting durchgeführt wurden, was im klinischen Alltag nahezu nicht umsetzbar erscheint. Welchen Effekt eine Gruppenintervention hat, ist bislang nur aus schmerztherapeutischen Interventionen im Rahmen der Behandlung eines chronischen Schmerzes bekannt. V.a. die positiven sozialen Aspekte einer Gruppentherapie können von der Autorin der vorliegenden Arbeit durch Erfahrungen im klinischen Alltag bestätigt werden. Vor dem Gesichtspunkt eines biopsychosozialen Ansatzes wäre eine präoperative PNE im Gruppensetting somit ein sinnvoller Ansatz [6].

\section{Fazit für die Praxis}

- "Pain neuroscience education“ (PNE) in einem präoperativen Setting kann Kinesiophobie, Angst und Katastrophisieren reduzieren. Der Effekt einer präoperativen PNE kann weniger an den Variablen Schmerz und Funktion bewertet werden, sondern eher durch psychometrische Assessments wie z. B. TSK und PCS.

Die Inhalte von PNE basieren auf der Neurobiologie und Ätiologie von Schmerzen sowie von zu Schmerz beitragenden Risikofaktoren.

- PNE hat einen systematischen Aufbau und kann einfach in die tägliche Praxis integriert werden.

\section{Korrespondenzadresse}

Prof. Dr. Harry von Piekartz, Ph.D., M.Sc. PT Fakultät Wirtschafts-und Sozialwissenschaften, Physiotherapie und Rehabilitationswissenschaften, Hochschule Osnabrück Caprivistr. 30a, 49076 Osnabrück, Deutschland H.von-Piekartz@hs-osnabrueck.de

Funding. Open Access funding enabled and organized by Projekt DEAL. 


\section{Einhaltung ethischer Richtlinien}

Interessenkonflikt. K. von Korn, T. Weiss und H. von Piekartz geben an, dass kein Interessenkonflikt besteht.

Für diesen Beitrag wurden von den Autoren keine Studien an Menschen oder Tieren durchgeführt. Für die aufgeführten Studien gelten die jeweils dort angegebenen ethischen Richtlinien.

Open Access. Dieser Artikel wird unter der Creative Commons Namensnennung 4.0 International Lizenz veröffentlicht, welche die Nutzung, Vervielfältigung, Bearbeitung, Verbreitung und Wiedergabe in jeglichem Medium und Format erlaubt, sofern Sie den/die ursprünglichen Autor(en) und die Quelle ordnungsgemäß nennen, einen Link zur Creative Commons Lizenz beifügen und angeben, ob Änderungen vorgenommen wurden.

Die in diesem Artikel enthaltenen Bilder und sonstiges Drittmaterial unterliegen ebenfalls der genannten Creative Commons Lizenz, sofern sich aus der Abbildungslegende nichts anderes ergibt. Sofern das betreffende Material nicht unter der genannten Creative Commons Lizenz steht und die betreffende Handlung nicht nach gesetzlichen Vorschriften erlaubt ist, ist für die oben aufgeführten Weiterverwendungen des Materials die Einwilligung des jeweiligen Rechteinhabers einzuholen.

Weitere Details zur Lizenz entnehmen Sie bitte der Lizenzinformation auf http://creativecommons.org/ licenses/by/4.0/deed.de.

\section{Literatur}

\section{Verwendete Literatur}

1. Apkarian AV, Bushnell MC, Treede R-D, Zubieta J-K (2005) Human brain mechanisms of pain perception and regulation in health and disease. Eur J Pain 9(4):463-484. https://doi.org/10.1016/j. ejpain.2004.11.001

2. Archer KR, Seebach CL, Mathis SL, Riley LH, Wegener ST (2014) Early postoperative fear of movement predicts pain, disability, and physical health six months after spinal surgery for degenerative conditions. Spine J 14(5):759-767

3. Butler DS (Hrsg) (2009) Schmerzen verstehen (Unter Mitarbeit von Moseley GL)

4. Chambel SS, Tavares I, Cruz CD (2020) Chronic pain after spinal cord injury: is there a role for neuronimmune dysregulation? Front Physiol 11:748. https://doi.org/10.3389/fphys.2020.00748

5. Fenton BW, Elim S, Zolton J (2015) The neurobiology of pain perception in normal and persistent pain. Pain Manag 5(4):297-317. https://doi.org/ 10.2217/pmt.15.27

6. Goudman L, Huysmans E, IckmansK, N Jo, Moens M, Putman Ket al (2019) A modern pain neuroscience approach in patients undergoing surgery for lumbar radiculopathy: A clinical perspective. Physical therapy 99(7):933-945. https://doi.org/ 10.1093/ptj/pzz053

7. Hildebrandt J (2002) B2 Rücken- und Halswirbelsäulenschmerzen. In: Diener HC, Maier C (Hrsg) Das Schmerz Therapie Buch - medikamentös, interventionell, psychologisch. Urban \& Fischer, München, S68-89

\section{Effects of preoperative neurobiological education on postoperative outcome. A systematic review}

Background: Pain may have a crucial impact on human quality of life. An increase in knowledge about neurobiological and neuroscientific processes alone can positively influence the subjective perception of pain as well as psychometric variables. There are different forms of preoperative patient education with the aim to explain postoperative pain. Based on current literature, preoperative biomedical education has a low level of evidence. It can increase the preoperative anxiety and stress level of patients, which has a negative impact on the postoperative outcome. In contrast, the neuroscientific understanding considers postoperative pain from the viewpoints of the plasticity of the nervous system and involves sensitizational processes in the central and peripheral nervous systems.

Purpose: To systematically investigate short- and long-term effects of pain neuroscience education (PNE) in patients before spine surgery.

Materials and methods: The literature search involved a search of medical databases according to the $\mathrm{PI}(\mathrm{C}) \mathrm{O}$ scheme, and 83 articles were shortlisted. Nine articles that met the inclusion and exclusion criteria were finally included.

Results: Preoperative pain neuroscience education can positively influence postoperative catastrophizing tendencies as well as postoperative kinesiophobia but has no influence on postoperative pain and function.

Conclusion: Preoperative reduction of anxiety and pain-maintaining factors mainly on the psychological and social level may have a positive effect on postoperative subjective pain evaluation, which is reflected in a reduction of anxiety, catastrophizing tendencies, and a lower utilization of postoperative health care services.

\section{Keywords}

Preoperative pain neuroscience education - Spinal surgery · Anxiety reduction · Catastrophizing · Postoperative chronification

8. Ickmans K, Moens M, Putman K, BuylR, Goudman L, Huysmans E et al (2016) Back school or brain school for patients undergoing surgery for lumbar radiculopathy? Protocol for a randomised, controlled trial. J Physiother 62(3):165

9. Kucyi A, Davis KD (2015) The dynamic pain connectome. Trends Neurosci38(2):86-95.https:// doi.org/10.1016/j.tins.2014.11.006

10. Lee C-H, Liu J-T, Lin S-C, Hsu T-Y, Lin C-Y, LongYau L (2018) Effects of educational intervention on state anxiety and pain in people undergoing spinal surgery: a randomized controlled trial. Pain Manag Nurs 19(2):163-171

11. Lluch E, Dueñas L, Falla D, Baert I, Meeus M, Sánchez-Frutos J, Nijs J (2018) Preoperative pain neuroscience education combined with knee joint mobilization for knee osteoarthritis: a randomized controlled trial. Clin J Pain 34(1):44-52. https://doi. org/10.1097/AJP.0000000000000511

12. Louw A (Hrsg) (2012) Your nerves are having back surgery, 1. Aufl. Orthopedic Physical Therapy Products,

13. Louw A, Diener I, Landers MR, Puentedura EJ (2014) Preoperative pain neuroscience education for lumbar radiculopathy: a multicenter randomized controlled trial with 1-year follow-up. Spine 39(18):1449-1457

14. Louw A, Diener I, Puentedura EJ (2015) The short term effects of preoperative neuroscience education for lumbar radiculopathy: a case series. Int J Spine Surg 9:11

15. Louw A, Puentedura EJ, Diener I, Peoples RR (2015) Preoperative therapeutic neuroscience education for lumbar radiculopathy: a single-case fMRI report. Physiother Theory Pract 31(7):496-508

16. Louw A, Diener I, Landers MR, Zimney K, Puentedura EJ (2016) Three-year follow-up of a randomized controlled trial comparing preoperative neuroscience education for patients undergoing surgery for lumbar radiculopathy. JSpine Surg 2(4):289-298

17. Louw A, Schmidt S, Puentedura E, Zimney K (2018) Pain neuroscience Education. Teaching People About Pain. 2nd Edition. Minneapolis: Orthopedic Physical Therapy Products.

18. Louw A, Farrell K, Choffin B, Foster B, Lunde G, Snodgrass $M$ et al (2019) Immediate effect of pain neuroscience education for recent onset low back pain: an exploratory single arm trial. J Man Manip Ther 27(5):267-276. https://doi.org/10. 1080/10669817.2019.1624006

19. Louw A, Rico D, Langerwerf L, Maiers N, Diener I, Cox T (2020) Preoperative pain neuroscience education for shoulder surgery: A case series. South Afr J Physiother. https://doi.org/10.4102/ sajp.v76i1.1417

20. Lucas AJ (2012) Failed back surgery syndrome: whose failure? Time to discard a redundant term. Br J Pain 6(4):162-165. https://doi.org/10.1177/ 2049463712466517

21. Lumley MA, Cohen JL, Borszcz GS, Cano A, Radcliffe AM, Porter LS, Schubiner H, Keefe FJ (2011) Pain and emotion: a biopsychosocial review of recent research. J Clin Psychol 67(9):942-968. https://doi.org/10.1002/jclp.20816

22. MalflietA, Kregel J, Meeus M, Roussel N, Danneels L, Cagnie B et al (2018) Blended-learning pain 
neuroscience education for people with chronic spinal pain: randomized controlled multicenter trial. Phys Ther 98(5):357-368. https://doi.org/10. 1093/ptj/pzx092

23. Melzack R (2001) Pain and the neuromatrix in the brain. J DentEduc 65(12):1378-1382

24. Moseley GL (2004) Evidence for a direct relationship between cognitive and physical change during an education intervention in people with chronic low back pain. Eur J Pain. https://doi.org/ 10.1016/S1090-3801(03)00063-6

25. Núñez-Cortés R, Espinoza-Ordóñez C, Pommer PP, Horment-Lara G, Pérez-Alenda S, CruzMontecinos C (2019) A single preoperative pain neuroscience education: is it an effective strategy for patients with carpal tunnel syndrome? Med Hypotheses 126:46-50. https://doi.org/10.1016/j. mehy.2019.03.013

26. Rhodes L, Nash C, Moisan A, Scott DC, Barkoh K, Warner WC et al (2015) Does preoperative orientation and education alleviate anxiety in posterior spinal fusion patients? A prospective, randomized study. J Pediatr Orthop 35(3):276-279

27. Rolving N, Oestergaard Gregersen L, Willert MV, Christensen FB, Blumensaat F, Bünger C, Nielsen CV (2014) Description and design considerations of a randomized clinical trial investigating the effect of a multidisciplinary cognitive-behavioural intervention for patients undergoing lumbar spinal fusion surgery. BMC Musculoskelet Disord 15:62

28. Vlaeyen JW, Linton SJ (2000) Fear-avoidance and its consequences in chronic musculoskeletal pain: a state of the art. Pain 85(3):317-332

29. Voorhies RM, Xiaozhang J, Najeeb T (2007) Predicting outcome in the surgical treatment of lumbar radiculopathy using the Pain Drawing Score, McGill Short Form Pain Questionnaire, and risk factors including psychosocial issues and axial joint pain. Spine J 7(5):516-524. https://doi.org/ 10.1016/j.spinee.2006.10.013

30. Waddell G, Burton AK, Main CJ (2003) Screening to Identify People at Risk of Long-term Incapacity for Work. A Conceptual and Scientific Review. Royal Society of Medicine Press, London

\section{Weiterführende Literatur}

31. Louw A, Butler DS, Diener I, Puentedura EJ (2013) Development of a preoperative neuroscience educational program for patients with lumbar radiculopathy. Am J Phys Med Rehabil 92(5):446-452

32. Moseley GL, Butler D (2017) Explain pain supercharged. The clinican's handbook. Noigroup

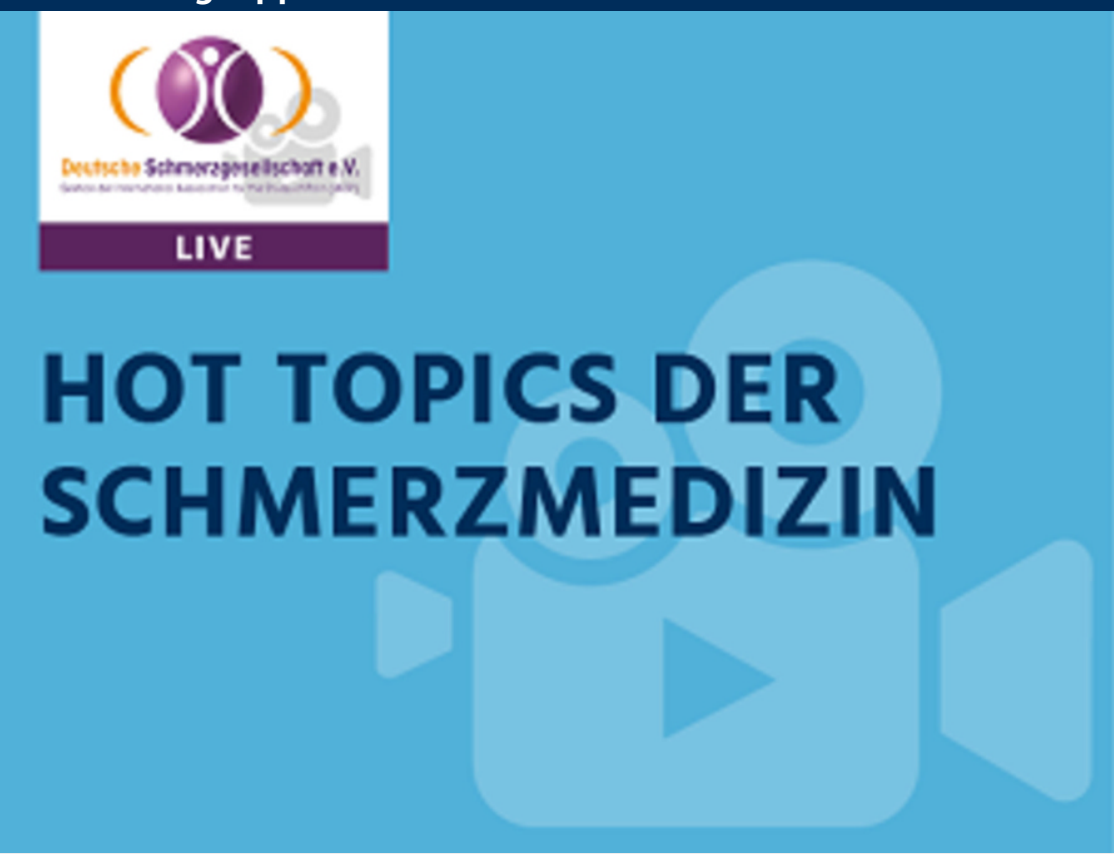

\section{Webinar-Reihe „Hot Topics der Schmerzmedizin“}

In der Webinar-Reihe „Hot Topics der Schmerzmedizin“ beleuchten wir regelmäßig aktuelle schmerzmedizinische Themen mit Expert*innen aus verschiedenen Fachgebieten. Als Teilnehmer*innen können Sie mitdiskutieren, indem Sie Ihre Fragen an die Expert*innen im Chat stellen. Die Teilnahme an den Webinaren ist nach Registrierung kostenfrei. Informieren Sie sich über die nächsten Termine und melden Sie sich an:

www.springermedizin.de/webinare-schmerzmedizin/18592486

- 19.01.23: Das ABC der Tumorschmerztherapie in der Praxis

Folgende Themen stehen on demand für Sie bereit:

- Das schmerzende lliosakralgelenk

- Schmerzchronifizierung verhindern - Wie funktioniert erfolgreiche Prävention?

- Opioide in der schmerzmedizinischen Praxis - S3-Leitlinie LONTS

- Kopfschmerzen und Migräne

- Schmerz bei rheumatischen Erkrankungen

- Achtsamkeit und Bewältigung chronischer Schmerzen

- Arthroseschmerz

- Cannabinoide in der Schmerztherapie - gezielt und verantwortungsvoll einsetzen

- Herausforderung chronische neuropathische Schmerzen - Diagnostik, Therapie, Prävention

- Nichtopiodanalgetika: Pharmakologie trifft Klinik

- Volkskrankheit Rückenschmerz - Multimodale Therapieansätze und Prävention in der Praxis

Die Webinar-Reihe "Hot Topics der Schmerzmedizin“ wird produziert von Springer Medizin und der Deutschen Schmerzgesellschaft e.V.

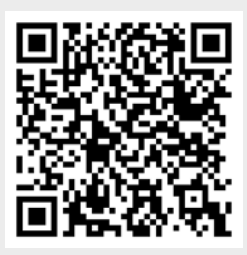


Hier steht eine Anzeige.

黑 Springer 